\title{
Data-driven criteria for defining SpA using MRI
}

Radiographic evidence of structural changes is often absent during the early stages of spondyloarthritis (SpA). Previous guidelines defining MRI-based criteria for SpA using the sacroiliac joint (SIJ) have been based on consensus, rather than on data. A data-driven definition of an SpA-positive SIJ using MRI has now been developed: preliminary evaluation of this measure indicates that evidence of bone marrow oedema in two SIJ quadrants, or erosion in one, is required for classification of axial SpA (axSpA).

Patients were recruited using two different strategies: in group A, back pain was the primary cause of referral (69 patients); in group B, patients had acute anterior uveitis and a history of back pain (88 patients). Participants were then classified by clinical examination and pelvic radiography as having nonradiographic SpA, ankylosing spondylitis, or nonspecific back pain.

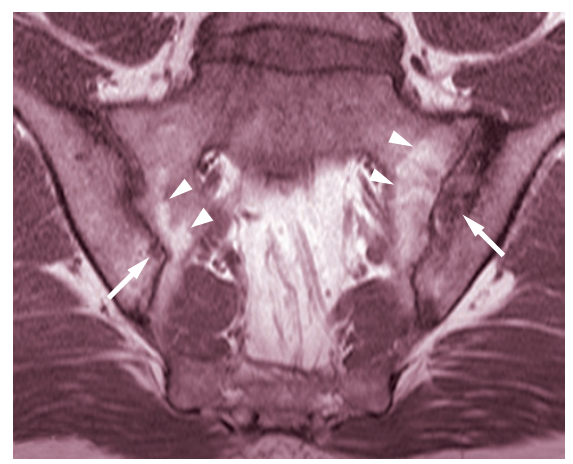

MRI image of the sacroiliac joint showing joint erosion (arrows) and fat infiltration (arrowheads). NPG

MRI images were evaluated by four independent readers. Each MRI scan, along with scans from 20 age-matched healthy control patients, was assessed globally for evidence of $\mathrm{SpA}$, and each SIJ quadrant was assessed for oedema, joint erosion, marrow fat infiltration, and ankylosis. Readers rated their confidence in the diagnosis of SpA on a scale of 1-10; scans with scores of 8-10 were considered to be SpA-positive. The scores were used to determine which characteristics had the highest correlation with clinical diagnosis.

The combined assessment of oedema and joint erosion reduced inter-reader variability and correlated best with clinical diagnosis. "Bone marrow oedema is observed in $20-30 \%$ of controls, which renders it an unsuitable feature to differentiate between cases and controls," says lead author Ulrich Weber. He highlights that global analysis for SpA, without analysis for specific lesions, was the best approach. "Only prospective data will tell whether a clinical or an MRI-based definition is preferable for classification of nonradiographic SpA."

Megan Cully

Original article Weber, U. et al. Development and validation of an MRI reference criterion for defining a positive SIJ MRI in spondyloarthritis. Arthritis Care Res. (Hoboken) doi:10.1002/acr.21893 